\title{
Numerical Analysis of Impedance Spectra of Yeast Suspensions
}

\author{
Ramos Airton, Farias D. Heric ${ }^{\dagger}$, \\ Electrical Engineering Department \\ Center of Technological Sciences \\ State University of Santa Catarina - Brazil \\ *airton_ramos@joinville.udesc.br, ${ }^{\dagger}$ hericdf@gmail.com
}

\begin{abstract}
Analysis of impedance spectrum of electroporated biological cell suspensions allows evaluating the state of permeabilization produced by the applied electric field. In this article we used a genetic algorithm to determine parameters of a proposed model for the impedance of suspension adjusting them numerically in order to minimize the error in relation to experimental spectra of Kluyveromyces marxianus CBS 6556 yeasts suspended in distilled water. It was possible to determine the variation of conductivity of suspension avoiding the influence of electrode polarization and dielectric dispersion due to interfacial and diffusion polarization on the cell surface. It was observed that the conductivity in the range of 1 to $100 \mathrm{KHz}$ increases 2.5 times with pulses of $400 \mathrm{KV} / \mathrm{m}$.
\end{abstract}

Index Terms - Electroporation, Genetic Algorithm, impedance spectrum, yeast cell suspension.

\section{INTRODUCTION}

Electrical impedance measurement of biological cell suspensions can be used to obtain information about concentration and size of the cells, internal and external conductivity and electrical properties of the cell membrane. The early studies on electrical properties of biological material date from the beginning of the last century, but the first revision probably was written in 1950 by H. P. Schwan. An extensive review on the main models and experimental results was compiled by Foster and Schwan in $1995[1]$.

One of the main difficulties in dispersion spectra analysis of biological material is the complexity of the models. Even for spherical cells suspended in a homogeneous conductor, the equations based on Maxwell-Wagner model have many parameters such as conductivity and dielectric constant of internal and external media, cell diameter, cell concentration, capacitance and conductance of the cell membrane. These parameters relate in a complex way to determine the dielectric response of the suspension. Dispersion equations in this case, provided in the revision of Foster and Schwan [1], describe a first order process. Moreover, when analyzing experimental results, the complexity becomes greater due to the influence of electrode polarization which contributes significantly to the total reactance at low frequencies [2].

An alternative approach for analyzing impedance spectra of cell suspensions is based on the use of 
numerical optimization methods, such as genetic algorithms [3]. With these methods it is possible with only moderate programming effort and small processing time, determine the values of various parameters used to fit the models with minimum error to impedance spectra obtained experimentally.

Electroporation is the process of pore openings in biological membranes by applying intense electric field in cell suspensions or biological tissues [4]-[7]. In the permeabilized state, the permeability and the conductivity of the cell membranes are increased resulting in higher conductivity of the medium [8],[9]. Although the pore opening process still remains not completely understood, it has been used as an important technique for tumor treatment, DNA and other macromolecules transfection [7],[10]-[12]. Nowadays electroporation process also has been applicable to enzyme extraction from yeast cells where this method proved to be more efficient than mechanical and chemical lyses [13]-[15].

The aim of this paper is to present experimental results and numerical analysis of impedance spectra of electroporated yeast suspensions obtained in different conditions of stimulation and using a genetic algorithm for model parameters determination.

\section{METHODS}

Yeast cells of Kluyveromyces marxianus CBS 6556 were grown aerobically in shaken flasks containing $0,15 \mathrm{~L}$ of culture medium (1\% yeast extract, $2 \%$ bactopeptone and $2 \%$ lactose) and incubated on an orbital shaker at $150 \mathrm{rpm}, 30^{\circ} \mathrm{C}$ for $12 \mathrm{~h}$. After reaching the stationary phase the colonies were centrifuged for six minutes at 12,000 rpm in an Eppendorf centrifuge and the supernatant was re-suspended in distilled water. This process is repeated three times. Observations in an Olympus CX31 optical microscope indicated that cells are spheroidal with average diameter $9.74 \pm$ $2.14 \mu \mathrm{m}$.

The electroporator consists basically of an arbitrary waveform generator implemented in a LabView program commanding a PCI 6251 board from National Instruments and an amplification stage to obtain up to $500 \mathrm{~V}$ and $5 \mathrm{~A}$ (short pulses up to $20 \mathrm{~ms}$ ) with balanced differential outputs. The signals are generated with 16-bit resolution and sampling frequency of $500 \mathrm{KHz}$. Figure 1 shows a schematic of the electroporation experiment. The sample holder consists of two cylindrical steel electrodes of $0.02 \mathrm{~m}$ diameter mounted on a nylon tube that keeps the surfaces of electrodes separated by $0.001 \mathrm{~m}$. All experiments were carried out at $25^{\circ} \mathrm{C}$. The generated signals where rectangular pulses with 1 to $10 \mathrm{~ms}$ duration and amplitude of 100 to $400 \mathrm{~V}$.

Electrical impedance in the range $40 \mathrm{~Hz}$ to $40 \mathrm{MHz}$ was measured on a 4294A Agilent Technologies impedance analyzer. The samples were measured immediately prior to the application of the electric field pulse and the measurement was repeated one minute after this application. This is the time required to disconnect the sample holder of the electroporator and connect it to the impedance analyzer as well as wait until the impedance reading stabilize.

The dielectric dispersion in a homogeneous suspension of cells can be modeled as a first-order 


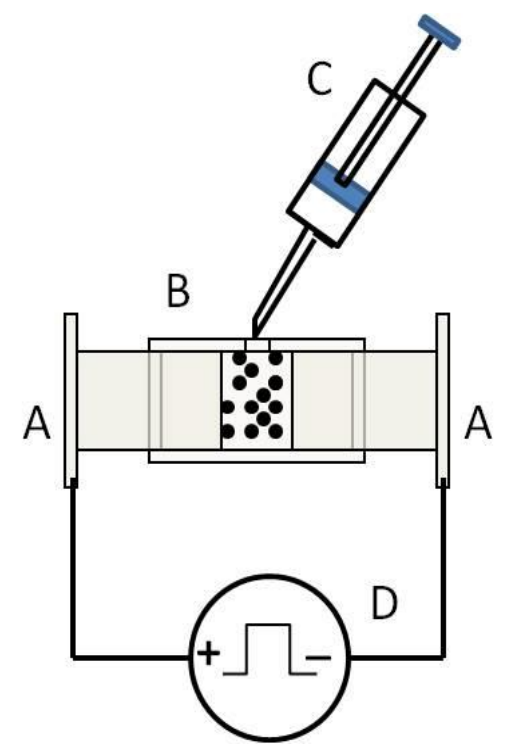

Fig. 1. Schematic representation of the electroporation experiment. (A) Steel electrodes, (B) Nylon support, (C) Syringe containing the cell suspension, (D) Pulsed voltage source.

relaxation process [1]. The equations (1) to (3) below show how the conductivity and dielectric constant depends on the frequency and how the amplitudes of dispersion $\left(\Delta \sigma\right.$ and $\left.\Delta \varepsilon_{r}\right)$ relate to the relaxation time $\left(\tau_{m}\right)$.

$$
\begin{gathered}
\sigma(\omega)=\sigma_{s}+\frac{\omega^{2} \tau_{m}^{2} \Delta \sigma}{1+\omega^{2} \tau_{m}^{2}} \\
\varepsilon_{r}(\omega)=\varepsilon_{\infty}+\frac{\Delta \varepsilon_{r}}{1+\omega^{2} \tau_{m}^{2}} \\
\Delta \sigma=\frac{\varepsilon_{o} \Delta \varepsilon_{r}}{\tau_{m}}
\end{gathered}
$$

In these equations $\sigma_{s}$ is the low frequency conductivity and $\varepsilon_{\infty}$ is the high frequency dielectric constant. By considering the geometry of the electrodes with parallel faces and small spacing/diameter ratio, the equation (4) below represents the sample impedance as the sum of the suspension impedance with the contact impedance with the metallic electrodes.

$$
Z_{m}=\frac{R_{c t}}{1+\left(j \omega \tau_{s}\right)^{\beta}}+\frac{d / A}{\sigma+j \omega \varepsilon_{r} \varepsilon_{o}}
$$

Where $d$ and $A$ are the distance and the area of the electrodes, respectively. The first term is the electrode-electrolyte interface impedance model according to McAdams et al [2], where $R_{c t}$ is named charge transfer resistance, $\beta$ is a constant and $\tau_{s}$ is the relaxation time for the process of charge transfer at the interface. The second term is the impedance of the cell suspension, in which the conductivity and dielectric constant are defined in equations (1) to (3). The numerical analysis of experimental impedance spectra according to the model described by equations (1) to (4) seeks to determine seven parameters: $R_{c t}, \beta, \tau_{s}, \sigma_{s}, \varepsilon_{\infty}, \Delta \varepsilon_{r}$ and $\tau_{m}$.

The method used to the parameterization of the impedance model is based on a Genetic Algorithm, which is a stochastic search technique based on natural selection and natural genetic mechanisms [3]. 
The program was developed with the following main components: (a) an initial population of individuals consisting of associations of model's parameters in a pre-defined sequence. This initial population is generated randomly; (b) a mechanism to appraise the response quality of each individual of the population. This mechanism was based on the quadratic medium error; (c) a method for joining fragments of the best individuals to form new ones in an attempt to obtain better individuals; (d) a mutation operator to avoid permanent loss of diversity of individuals. This intrinsic generality of the algorithm is responsible to its applicability to an extremely wide range of problems. We used 32-bit unsigned integer to linearly encode each of the parameters. The size of the population was 100 individuals. The selection was carried out by "Russian roulette" with elitism [3] and the bit mutation probability was fixed at $0.47 \%$ (equivalent to an average of 1.2 mutated bits per individual). Search ranges for each parameter were estimated on a simplified prior analysis of spectra. The program was written in C\# language and was executed in Windows 7 operating system microcomputer with Core 2 Duo processor running at $3 \mathrm{GHz}$ and $4 \mathrm{~GB}$ of RAM memory. Each simulation took about $100 \mathrm{~ms}$ to converge with an error less than or equal to $1 \%$. The error is obtained as the average squared difference between numerical values and experimental values by taking as a reference the quadratic mean of the experimental values.

\section{RESULTS}

The electroporation assays and impedance measurement were made with three different suspensions with average volume fraction of cells around 3.3\% obtained using the relationship between $\sigma_{o}$ and $\Delta \sigma$ provided in the study by Ramos and Schneider [15]. For each suspension were performed twenty-four assays with four field intensities (100, 200, 300 and $400 \mathrm{KV} / \mathrm{m})$ and six time intervals $(1,2,4,6,8$ and $10 \mathrm{~ms})$. Figure 2 shows the impedance spectrum obtained for a sample submitted to $400 \mathrm{KV} / \mathrm{m}$ for $10 \mathrm{~ms}$ compared to the spectrum of the intact suspension. The points in this figure correspond to the analytical model described in equation (4) whose numerically adjusted parameters are shown in the figure caption. Sample impedance variations below $1 \mathrm{KHz}$ are determined by electrode polarization effects where the reactance and resistance increases as the frequency decreases. However, above $1 \mathrm{KHz}$ the sample impedance is determined by the properties of the cell suspension. Between $1 \mathrm{KHz}$ and $100 \mathrm{KHz}$ the reactance is very low and the resistance approximately independent of frequency. The resistance is high because the ionic current circulates mainly on the external medium since the plasma membrane partially blocks the entry of ions in cells. The main dielectric dispersion band occurs between $100 \mathrm{KHz}$ and $1 \mathrm{MHz}$ being due to interfacial polarization on the surface of cell membranes [1]. The dispersion in impedance spectrum is perceived by a little more intense reduction in resistance than would be expected by the normal process of relaxation of the conductive medium. In the electroporated sample the resistance decreases significantly between 1 and $100 \mathrm{KHz}$ for about a third of the value prior to electroporation. This is caused by the opening of pores in the plasma membrane of cells, allowing the movement of ions 


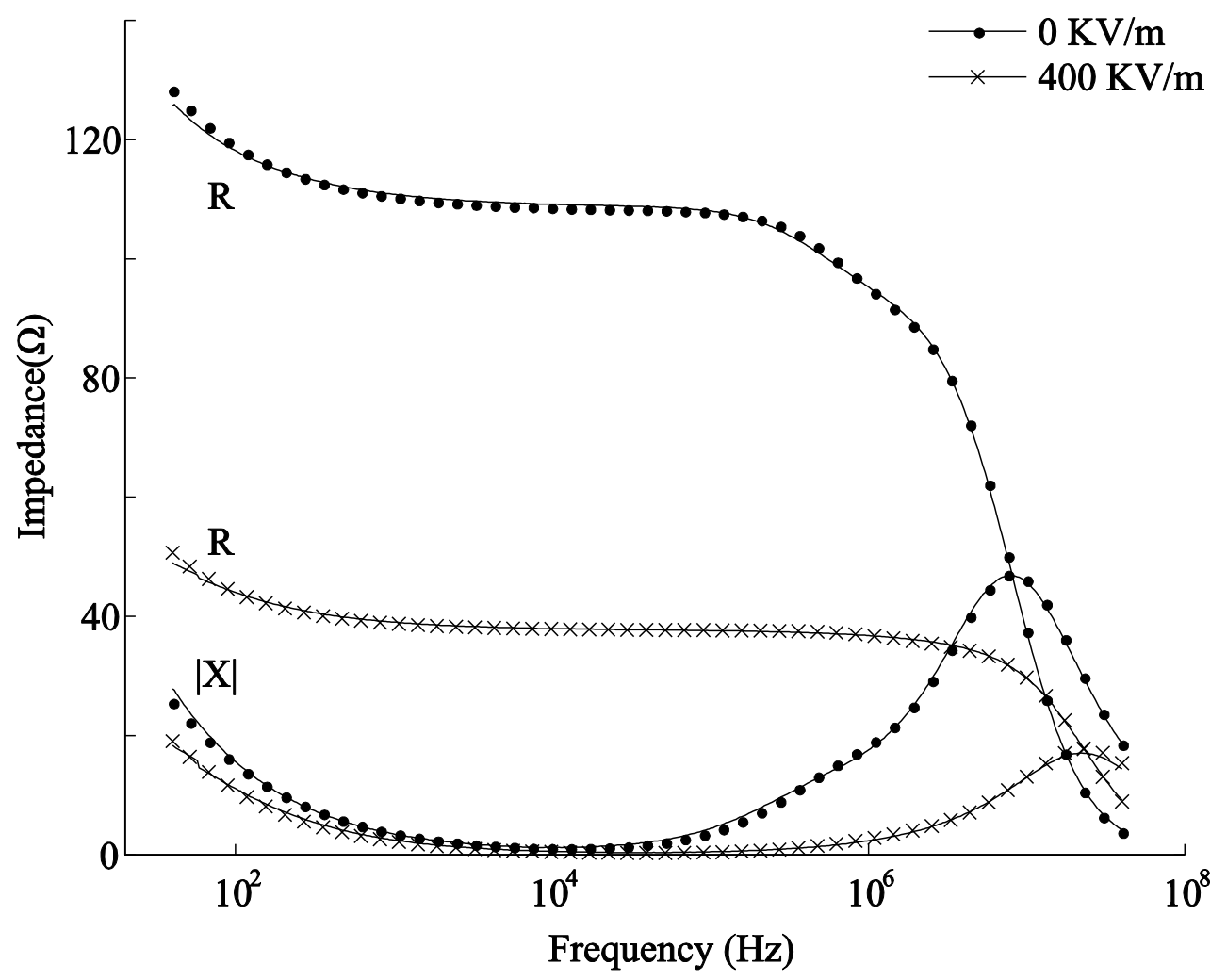

Fig. 2. Impedance spectrum of yeast suspension before and after applying the $400 \mathrm{KV} / \mathrm{m}$ field during $10 \mathrm{~ms}$. $\mathrm{R}$ - resistance, $|\mathrm{X}|$ - reactance module. The numerically obtained parameters used to plot points are: $0 \mathrm{KV} / \mathrm{m}: \mathrm{R}_{\mathrm{ct}}=220 \Omega, \beta=0.66, \tau_{\mathrm{s}}=64$ $\mathrm{ms}, \sigma_{\mathrm{s}}=30 \mathrm{mS} / \mathrm{m}, \Delta \varepsilon_{\mathrm{r}}=108, \tau_{\mathrm{m}}=0.21 \mu \mathrm{s}$ and $\varepsilon_{\infty}=75 ; 400 \mathrm{KV} / \mathrm{m}: \mathrm{R}_{\mathrm{ct}}=142 \Omega, \beta=0,71, \tau_{\mathrm{s}}=45 \mathrm{~ms}, \sigma_{\mathrm{s}}=85 \mathrm{mS} / \mathrm{m}, \Delta \varepsilon_{\mathrm{r}}=$ $60, \tau_{\mathrm{m}}=0.10 \mu \mathrm{s}$ and $\varepsilon_{\infty}=70$.

through intracellular medium, this way increasing the conductance of the suspension.

Table 1 presents the impedance model parameters obtained for all assays. The column named "Zero" relates the mean values and standard deviations for the parameters of the seventy-two samples measured prior to the application of the electric field. The columns for the applied field provide the average of eighteen values corresponding to six time intervals for each of the three suspensions. In the model of the electrode interface impedance the parameter that presents greater variation with the applied field is the charge transfer resistance. Probably the increase in resistance is due to the accumulation of products of reactions that occur on the surface of the electrodes. After each electroporation assay, the electrodes were cleaned and polished to remove the deposited layer. With the increase of the field it was observed that the deposited layer was darker indicating greater thickness. This layer possibly decreases the electric charge transfer between suspension and electrodes. The parameter of the suspension impedance more intensely affected by electroporation is the conductivity at low frequencies. The conductivity after application of $400 \mathrm{KV} / \mathrm{m}$ is greater than twice the intact suspension's value. This is a direct consequence of pore opening in the plasma membrane of cells. The moving ions in suspension can cross cell membranes through these pores. In addition, some ions from the internal medium diffuse out cells through the pores, increasing the ion concentration and conductivity of the external medium. In an intact suspension, ions accumulate in the inner and outer faces of the membranes, producing the effect of interfacial polarization and 
TABLE I. DISPERSION MODEL PARAMETERS BEFORE AND AFTER APPLICATION OF ELECTRIC FIELD

\begin{tabular}{cccccc}
\hline \multirow{2}{*}{ Parameter } & \multicolumn{5}{c}{ Electric Field $(\mathrm{KV} / \mathrm{m})$} \\
& Zero & $\mathbf{1 0 0}$ & $\mathbf{2 0 0}$ & $\mathbf{3 0 0}$ & $\mathbf{4 0 0}$ \\
\hline$R_{c t}(\Omega)$ & $140 \pm 50$ & 137 & 144 & 154 & 160 \\
$\beta$ & $0.72 \pm 0.09$ & 0.75 & 0.74 & 0.75 & 0.75 \\
$\tau_{s}(\mathrm{~ms})$ & $35 \pm 24$ & 34 & 37 & 39 & 35 \\
$\sigma_{s}(\mathrm{mS} / \mathrm{m})$ & $25 \pm 7$ & 30 & 31 & 48 & 62 \\
$\Delta \varepsilon_{r}$ & $143 \pm 49$ & 136 & 126 & 110 & 88 \\
$\tau_{m}(\mu \mathrm{s})$ & $0.34 \pm 0.1$ & 0.30 & 0.29 & 0.22 & 0.18 \\
$\varepsilon_{\infty}$ & $75 \pm 6$ & 77 & 73 & 74 & 74 \\
\hline
\end{tabular}

limiting conductivity at low frequencies, since the ionic current is forced to circumvent the cells. However, data in Table I show that the dielectric dispersion is reduced by the process of electroporation. Both the dielectric constant $\Delta \varepsilon_{r}$ and relaxation time $\tau_{m}$ decrease with increasing intensity of the applied field. The conduction through the pores decreases ion accumulation and consequently polarization of membranes.

Figure 3 shows the variation of conductivity at low frequencies in relation to the applied field strength and pulse duration. To $100 \mathrm{KV} / \mathrm{m}$ no significant increase of conductivity for any pulse duration occurs and to $200 \mathrm{KV} / \mathrm{m}$ an almost linear increase is observed. For higher field strengths the conductivity increases rapidly in one millisecond and after the increase occurs at a slower rate. This is because electroporation has a fast phase of hundreds of microseconds in which the pores are created followed by a slow phase in which the created pores increase in diameter [8], [9].

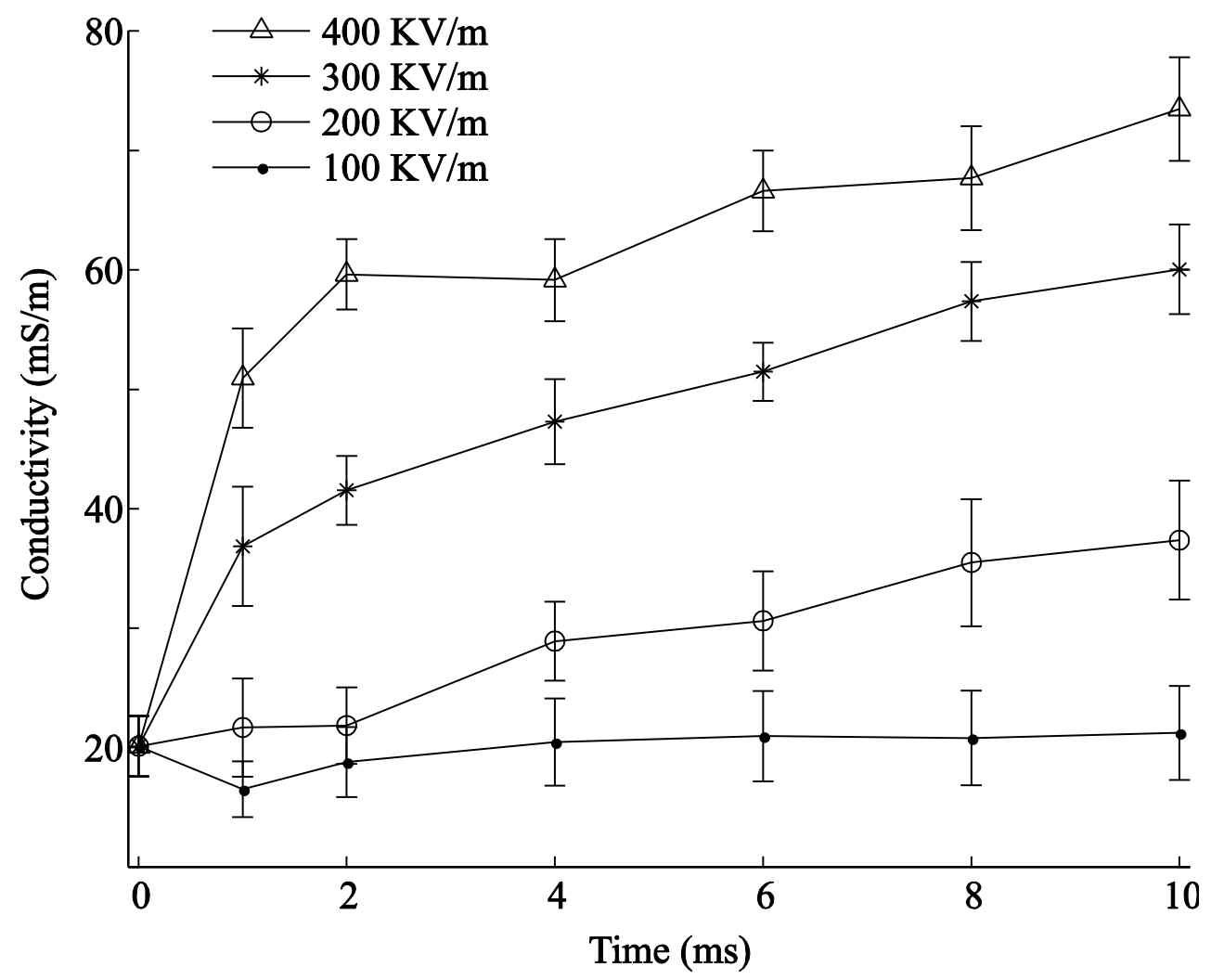

Fig. 3. Low-frequency conductivity of cell suspension as a function of the applied field strength and pulse duration. 


\section{DISCUSSION}

Numerical simulation based on genetic algorithm presents itself as an alternative of significant value in modeling complex systems described by mathematical models with unknown parameters. In the case of electrical impedance of the cell suspension, it is possible to isolate and identify processes that occur in the electrolyte, as conductivity at low frequencies and in the plasma membrane, as the interfacial polarization. In the characterization of cell electroporation, the presented numerical modeling allows obtaining the changes in electrical impedance parameters of the sample according to the applied electric field and the pulse duration.

The obtained results for the behavior of the conductivity and dielectric dispersion in the electroporation agree with models and experimental results already published [1], [8], [9]. Due to the opening of pores when subjected to intense electric fields, cell membranes allow passage of ions through lipid matrix. This process causes increase of suspension conductivity and reduction of interfacial polarization, which reduces the dielectric constant of the medium.

Conductivity measurements have been used in published works as an indicator of the intensity of the electroporation [16]-[20]. In these cases, sample conductivity was obtained through instantaneous values of electric current and potential difference in the sample during the application of the electric field. This method quite widespread among researchers of cell electroporation presents an important deficiency due to the lack of any compensation procedure of the electrode polarization and dielectric dispersion in the sample. Voltage pulses with hundreds of microseconds to milliseconds of duration exhibit a wide spectrum of frequencies. Hence, the current flowing in the sample can be strongly affected by the impedance of the electrode surface and dielectric dispersion due to interfacial polarization. In addition, during the pulse application, the heat caused by power dissipation increases additionally the electrolyte conductivity [15], [16].

The impedance measurement after the pulse and parameterization using the method presented in this article overcome these problems, so that the obtained conductivity is the correct value for the suspension in the intermediate frequency range of the spectrum. This value can be properly related to electroporation according to the models already proposed in the literature [1], [8], [9]. Recently, an alternative method for avoiding sample reactance interference in conductivity measurement during electroporation was proposed [21]. In this work, the conductivity of yeast cell suspensions was measured by means of a high frequency sinusoidal signal with small amplitude added to the voltage pulse. The small signal frequency was chosen in the frequency range where the sample reactance is very small [21].

\section{CONCLUSION}

The use of genetic algorithm in search of appropriate values to the parameters of the impedance model of yeast suspensions has shown to be efficient. Programming is simple and processing is fast. This approach allows assessing the changes in conductivity of suspension due to pore opening in the 
cell membrane, isolating the effects of electrode polarization and dielectric dispersion of the suspension. It was observed that the pulse of $400 \mathrm{KV} / \mathrm{m}$ increased the conductivity between 1 and 100 $\mathrm{KHz}$ at least 2.5 times compared to the intact suspension. The technique of impedance measurement after pulse application and analysis by using genetic algorithm is a powerful tool for study and characterization of cell electroporation.

\section{REFERENCES}

[1] K. R. Foster and H. P. Schwan "Dielectric properties of tissues," in Handbook of Biological Effects of Electromagnetic Fields, $2^{\text {nd }}$ ed., C. Polk and E. Postow, CRC, New York, 1995, pp. 25-102.

[2] E. T. McAdams, A. Lackermeier, J. A. McLaughlin, D. Macken and J. Jossinet, "The linear and non-linear electrical properties of the electrode-electrolyte interface," Biosensors and Bioelectronics, vol. 10, 1995, pp. 67-74.

[3] D. A. Coley, An Introduction to Genetic Algorithms for Scientists and Engineers, $1^{\text {st }}$ ed., World Scientific Publishing, Singapore, 1999.

[4] K. Kinosita and T. Y. Tsong, "Voltage-induced conductance in human erythrocyte membranes," Biochim. Biophys. Acta, vol. 554, pp. 479-497, Jul. 1979.

[5] D. C. Chang, "Structure and dynamics of electric field-induced membrane pores as revealed by rapid-freezing electron microscopy," in Guide to Electroporation and Electrofusion,D. C. Chang, B. M. Chassy, J. A. Saunders, and A. E. Sowers, Eds. San Diego, CA: Academic, 1991, pp. 9-27.

[6] J. Teissié, M. Golzio, and M. P. Rols, "Mechanisms of cell membrane electropermeabilization: A minireview of our present (lack of ?) knowledge," Biochim. Biophys. Acta, vol. 1724, pp. 270-280, Aug. 2005.

[7] D. Miklavčič and M. Puc, "Electroporation," in Wiley Encyclopedia of Biomedical Engineering. M. Akay, New York: Wiley, 2006, pp. 1-11.

[8] C. Chen, S. W. Smye, M. P. Robinson and J. A. Evans, "Membrane electroporation theories: a review," Medical and Biological Engineering and Computing, vol. 44, 2006, pp. 4-14.

[9] J. C. Weaver and Y. A. Chizmadzhev, "Theory of electroporation: a review," Bioelectrochemistry, vol. 41: pp. 135$160,1996$.

[10] S. D. Reed and S. Li, "Electroporation advances in large animals," Curr. Gene Ther, vol. 9, pp. 316-326, Aug. 2009.

[11] J. Rice, C. H. Ottensmeier, and F. K. Stevenson, "DNA vaccines: Precision tools for activating effective immunity against cancer," Nat. Rev. Cancer, vol. 8, pp. 108-120, Feb. 2008.

[12] M. Pavlin, M. Kanduser, M. Rebersek, G. Pucihar, F. X. Hart, F. R. Magjarevic, and D. Miklavcic, "Effect of cell electroporation on the conductivity of a cell suspension,” Biophys. J., vol. 88, pp. 4378-4390, Jun. 2005.

[13] V. Ganeva and B. Galutzov, "Electropulsation as an alternative method for protein extraction from yeast," FEMS Microbiology Letters, vol. 174, pp. 279-284, 1999.

[14] V. Ganeva, B. Galutzov, N. Eynard and J. Teissié, "Electroinduced extraction of $\beta$-galactosidase from Kluyveromyces lactis," Appl. Microbiol. Biotechnol., vol. 56, pp. 411-413, 2001.

[15] A. Ramos and A. L. Schneider, "Electroporation of Kluyveromyces marxianus and $\beta$-D-galactosidase extraction," in Ganesh R. N. Applied Biological Engineering - Principles and Practice, Ed. Intech, Melbourne, 2012.

[16] M. Pavlin, M. Kanduser, M. Rebersek, G. Pucihar, F. X. Hart, R. Magjarevic and D. Miklavcic, "Effect of cell electroporation on the conductivity of a cell suspension," Biophysical Journal, vol. 88, 2005, pp. 4378-4390.

[17] D. O. H. Suzuki, A. Ramos, M. C. M. Ribeiro, L. H. Cazarolli, F. R. M. B. Silva, L. D. Leite and J. L. B. Marques "Theoretical and experimental analysis of electroporated membrane conductance in cell suspension," IEEE Transactions on Biomedical Engineering, vol. 58, pp. 3310-3318, 2011.

[18] H. He, D. C. Chang, and Y. K. Lee, "Nonlinear current response of micro electroporation and resealing dynamics for human cancer cells," Bioelectrochemistry, vol. 72, pp. 161-168, Apr. 2008.

[19] M. Khine, C. Ionescu-Zanetti, A. Blatz, L. Wang, and L. P. Lee, "Single cell electroporation arrays with real-time monitoring and feedback control," Lab Chip, vol. 7, pp. 457-462, Mar. 2007.

[20] A. Ivorra, B. Al-Sakere, B. Rubinsky, and L. M. Mir, "In vivo electrical conductivity measurements during and after tumor electroporation: Conductivity changes reflect the treatment outcome," Phys. Med. Biol., vol. 54, pp. 5949-5963, Sep. 2009.

[21] A. Ramos, A. L. S. Schneider, D. O. H. Suzuki and J. L. B. Marques, "Sinusoidal Signal Analysis of Electroporation in Biological Cells,” IEEE Transactions on Biomedical Engineering, vol. 59, no. 10, pp. 2965-2973, Oct. 2012. 